\title{
Bipolar disorder, comorbid anxiety disorders, gynecomastia and dental pain: case analysis with literature review*
}

\author{
Kenneth R. Kaufman, Ronke Babalola, Miriam Campeas and Melissa Coluccio
}

Bipolar disorder with comorbid anxiety disorders frequently requires rational polypharmacy, including use of serotonergic psychotropics. These may result in adverse effects, influencing adherence, complicating treatment and confounding diagnoses. serotonergic non-adherence is associated with discontinuation syndromes. In this complex case with an on/off/on/off design, both dose-dependent buspirone-induced gynecomastia and buspirone discontinuation syndrome with dental pain are reported. Clinicians and patients should consider these findings to maximise treatment adherence, minimise any unnecessary interventions and address unusual adverse effects. Since patients may not voluntarily disclose specific adverse effects and often do not acknowledge non-adherence, clinician-directed questions are required. This case further emphasises the importance of medication and symptom timelines to guide determination of causation for adverse effects. Although findings from this case cannot be generalised, they suggest the need for continued clinician and patient education, as well as the benefit from detailed case reports

\section{Declaration of interest}

None.

\section{Copyright and usage}

(C) The Royal College of Psychiatrists 2018. This is an Open Access article, distributed under the terms of the Creative Commons Attribution-NonCommercial-NoDerivatives licence (http://creativecommons.org/licenses/by-nc-nd/4.0/), which permits noncommercial re-use, distribution, and reproduction in any medium, provided the original work is unaltered and is properly cited. The written permission of Cambridge University Press must be obtained for commercial re-use or in order to create a derivative work.
Bipolar disorder psychiatric comorbidities are important in determining diagnosis, treatment and outcome. Anxiety disorder comorbidity in bipolar disorder patients is often underappreciated by clinicians. Recent systematic reviews and meta-analyses have noted lifetime comorbidity prevalence rates of $42.7 \%$ for any anxiety disorder, $15.1 \%$ for generalised anxiety disorder (GAD), $10.8 \%$ for post-traumatic stress disorder (PTSD), $16.8 \%$ for panic disorder, and $17.0 \%$ for obsessive-compulsive disorder (OCD). ${ }^{1-3}$ Comorbid anxiety disorders may be associated with an earlier age at onset for bipolar disorder, ${ }^{4}$ as well as poorer acute treatment of the disorder and longitudinal course outcomes. ${ }^{5,6}$ Similarly, substance use disorders (SUD) are commonly comorbid with bipolar disorder (combining all SUD 35-40\%, with alcohol > cannabis > other illicit drugs $)^{7,8}$ and may be associated with early age at onset (child and adolescent populations) for bipolar disorder. ${ }^{4}$ SUD, in particular, cannabis use disorder (CUD), may also be associated with self-medication of comorbid anxiety disorders. ${ }^{9}$ Further, attention-deficit hyperactivity disorder (ADHD) has been reported in $>20 \%$ of patients with bipolar disorder and may be associated with earlier age at onset for bipolar disorder with poorer outcome. ${ }^{10}$

Treatment of bipolar disorders with comorbid anxiety disorders and SUD may be complex and often requires rational polypharmacy, including combined mood-stabilising agents (lithium, antiepileptic drugs and second-generation antipsychotics) and serotonergic psychotropics (selective serotonin reuptake inhibitors and serotonin-noradrenaline reuptake inhibitors). ${ }^{11,12}$ Optimal care for all medical conditions, and bipolar disorder with psychiatric comorbidities in particular, is premised on maximising therapeutic response and minimising adverse effects. ${ }^{13}$ The key to positive treatment outcomes in mood disorders is psychotropic adherence, which has frequently been reported to be $<50 \% .{ }^{14-18} \mathrm{~A}$ recent review emphasised that strategies for treatment adherence remain an unmet need for bipolar disorder. ${ }^{18}$

* Presented in part at the 19th Annual Conference of the International Society of Bipolar Disorders, Washington DC, May 4-7, 2017.
Adverse effects associated with serotonergic psychotropics influence general treatment adherence and psychotropic adherence, complicate treatment and may even confound diagnoses. In treating bipolar disorders with comorbid anxiety disorders, specific adverse effects may be related to both serotonergic agents and mood stabilisers, requiring an accurate timeline of psychotropic initiation, dosing and development of adverse effects to determine causation in the presence of polypharmacy. As adverse effects are a common factor leading to psychotropic non-adherence, consideration of all adverse effects is paramount to ensure adherence to pharmacotherapy. ${ }^{16}$ Often, specific adverse effects that might lead to non-adherence, including sexual dysfunction and mammoplasia or gynecomastia, are neither voluntarily reported by patients nor directly questioned by clinicians. ${ }^{19-22}$

When considering the influence of adverse effects on adherence and treatment outcome, it is also important to consider the effects of rapid or abrupt discontinuation of the purported offending agent and the development of psychotropic discontinuation syndromes, which can cause diagnostic dilemmas, treatment mismanagement and increased morbidity. ${ }^{23}$ Specifically, the antidepressant discontinuation syndrome associated with serotonergic agents includes a pot pourri of symptoms including, but not limited to, depression, anxiety, hallucinations, confusion, fatigue, tremors, paraesthesia, insomnia, dizziness, vertigo, vivid dreams, labile mood, irritability or anger, suicidal ideation, and hypomania or mania. ${ }^{23-25}$

This case report describes both a previously unreported discontinuation symptom (dental pain) and gynecomastia in a patient with bipolar disorder and multiple psychiatric comorbidities (GAD, panic disorder, PTSD, OCD, ADHD and SUD) treated with rational polypharmacy including serotonergic psychotropics.

\section{Method}

The study consisted of a case analysis with a PubMed literature review. 


\section{Results}

A 21-year-old male college student presented with a major depressive episode (MDE), GAD of sixth month duration, CUD, and alcohol use disorder (AUD). The patient was in excellent medical health and denied any historical or active medical diagnoses; his body mass index (BMI) was $23.14 \mathrm{~kg} / \mathrm{m}^{2}$. During the psychiatric evaluation, this patient acknowledged chronic depressive features since age 16, but masked other psychopathology beyond GAD, CUD and AUD. Initial psychotropic treatments included escitalopram $5 \mathrm{mg}$ q.a.m. and buspirone $7.5 \mathrm{mg}$ b.i.d. which were titrated to $20 \mathrm{mg}$ q.d. and $15 \mathrm{mg}$ t.i.d., respectively, with the addition of lorazepam $0.5 \mathrm{mg}$ b.i.d. p.r.n. to address situational exacerbation of the patient's anxiety. After a 4-month therapeutic escitalopram trial with persistent complete neurovegetative-affective cluster, bupropion $150 \mathrm{mg}$ q.a.m. was added to his psychotropic regimen.

Shortly thereafter, the patient abruptly decreased both escitalopram to $10 \mathrm{mg}$ q.a.m. and buspirone to $7.5 \mathrm{mg}$ t.i.d. for 2 days and described the following psychotropic discontinuation features - agitation, restlessness, fogginess, word-finding difficulties, memory loss, diaphoresis, diarrhoea, headache, shivering, goose bumps, fever and 'feeling glass shards in my bones'. To address the newonset discontinuation features, escitalopram was increased to $15 \mathrm{mg}$ q.a.m. followed by a gradual taper ( $2.5 \mathrm{mg} /$ week) with resolution of all discontinuation features over the course of 3 weeks. Buspirone was returned to the prior $15 \mathrm{mg}$ t.i.d. dosing. Further, the patient commented on using cannabis both to treat anxiety and to diminish the discontinuation features. At this time, the patient acknowledged panic attacks that were independent of situational stressors, and he was diagnosed with panic disorder without agoraphobia. Lorazepam was increased to $0.5-1 \mathrm{mg}$ t.i.d. p.r.n.. To better treat the patient's ongoing depression, bupropion was titrated to $200 \mathrm{mg}$ q.a.m. and then $300 \mathrm{mg}$ q.a.m..

All standard blood chemistries, including thyroid function tests, lipid profile and vitamin $\mathrm{D}$, were within normal limits; however, the patient's total testosterone level of $313 \mathrm{ng} / \mathrm{dL}$ (laboratory reference range $348-1197 \mathrm{ng} / \mathrm{dL}$ ) led to treatment with topical testosterone $50 \mathrm{mg} / 5 \mathrm{~g}$ gel q.a.m. on the right upper extremity. Within 2 weeks of initiation of testosterone and the increased bupropion, the patient reported new-onset left breast (contralateral to testosterone gel) gynecomastia with tenderness, which was confirmed by mammography - testosterone was discontinued with total treatment course of 3 weeks only. At the same session, the patient presented with continuing depressive features, as well as intermittent brief hypomanic/manic features. Historical bipolar features were reassessed, and he acknowledged not having previously shared their presence since age 13 - these brief hypomanic/manic features never lasted beyond 1-2 days. The patient's mood disorder diagnosis was changed to bipolar disorder not otherwise specified (NOS). Further, with acknowledgment of compulsive counting and folding from youth with obsessive thoughts, the patient was diagnosed with OCD. Lurasidone $40 \mathrm{mg}$ q. dinner was initiated to address his bipolar features, but discontinued within 2 days secondary to marked akathisia which responded to a brief course of benztropine mesylate $0.5 \mathrm{mg}$ b.i.d. p.r.n.. Subsequently, lithium $600 \mathrm{mg}$ q.h.s. was initiated but discontinued within 2 weeks secondary to polyuria, polydipsia and new-onset tremor.

Given the continuing depressive features and adverse effects with both lurasidone and lithium, alternative interventions for bipolar depression were reviewed; however, the patient initially declined both anti-epileptic drugs and other second-generation antipsychotics. As attention issues were a key concern to his studies, ADHD criteria were re-assessed. Whereas previously he denied meeting criteria for ADHD, he now admitted full criteria which dated back to elementary school and further commented that testing for ADHD had been recommended but had not been pursued. Amphetamine-dextroamphetamine extended release $10 \mathrm{mg}$ q.a.m. was initiated for the newly diagnosed ADHD. Since there was no reduction in gynecomastia with continuing tenderness and pain during the 3 weeks following testosterone and escitalopram discontinuation, buspirone was reduced to $15 \mathrm{mg}$ b.i.d..

With amphetamine-dextroamphetamine extended release titrated to $20 \mathrm{mg}$ q.a.m., ADHD further resolved. Aripiprazole $2 \mathrm{mg}$ q.h.s. was added to address limited paranoia and hypersexuality and to serve as an anxiolytic agent, permitting further reduction in buspirone to $7.5 \mathrm{mg}$ t.i.d.. When aripiprazole was increased to $4 \mathrm{mg}$ q.h.s., the patient presented with akathisia, which responded to a brief course of benztropine mesylate $0.5 \mathrm{mg}$ b.i.d. p.r.n., and aripiprazole was discontinued. Nonetheless, the patient gradually tapered buspirone over 1 month $(22.5 \mathrm{mg} / \mathrm{day}$, $20 \mathrm{mg} /$ day, $17.5 \mathrm{mg} /$ day and then $15 \mathrm{mg} /$ day). With buspirone tapering, the patient reported a dose-dependent resolution of gynecomastia; on buspirone $15 \mathrm{mg}$ q.d., the patient described very minimal gynecomastia - 'almost unnoticeable and no pain'. Further, during buspirone tapering, the patient reported recurrent discontinuation features, as previously described, and new-onset dental pain. A dental consultation with full assessment found no pathology, including bruxism, associated with this dental pain with non-response to antibiotics. On low-dose buspirone, significantly increased generalised anxiety was noted, and buspirone was directly increased to $15 \mathrm{mg}$ b.i.d.. Within 7-10 days, the patient reported increased unilateral gynecomastia with recurrent tenderness and pain ('breast silhouette with B-size cup'). With buspirone increased to $15 \mathrm{mg}$ b.i.d., all discontinuation features including dental pain resolved.

Although the patient noted improvement of multiple features on his combined bupropion $300 \mathrm{mg}$ q.a.m., amphetamine-dextroamphetamine extended release $20 \mathrm{mg}$ q.a.m., and buspirone $15 \mathrm{mg}$ b.i.d. regimen, lamotrigine was initiated to better treat his bipolar disorder. The patient responded well to lamotrigine slow titration with resolution of remaining depressive features. At this juncture, with continuing stabilisation, the patient acknowledged long-standing PTSD, which he felt could finally be addressed in his ongoing CBT (this patient was in therapy prior to and throughout treatment by the psychiatrist).

Concomitantly, the patient was evaluated by a breast surgical oncologist, who concluded that the patient had drug-induced gynecomastia. A repeat testosterone level was $413 \mathrm{ng} / \mathrm{dL}$. Buspirone was again titrated down to $15 \mathrm{mg}$ q.d. with minimal gynecomastia compared with higher doses and no tenderness; however, he also had recurrent discontinuation features and dental pain. Thereafter, buspirone was gradually discontinued $(10 \mathrm{mg} /$ day, $7.5 \mathrm{mg} /$ day, $5 \mathrm{mg} /$ day and $2.5 \mathrm{mg} /$ day). With each step, the patient noted dental pain which persisted for 1 week. Of note, the other discontinuation features lasted for several weeks after final titration. Off buspirone, the patient commented on subjective total resolution of gynecomastia; however, a repeat mammogram revealed minimal bilateral gynecomastia. The patient was again evaluated by the same breast oncologist, who noted no gynecomastia on physical examination and considered the current mammogram to be benign.

The patient remains stabilised with the following psychotropic regimen: bupropion $300 \mathrm{mg}$ q.a.m., amphetamine-dextroamphetamine extended release $20 \mathrm{mg}$ q.a.m., lamotrigine $150 \mathrm{mg}$ q.h.s. and lorazepam $1 \mathrm{mg}$ t.i.d. p.r.n.

\section{Discussion}

This unique case permits discussion of different aspects of patient symptom reporting, early-onset bipolar disorder with delayed 
treatment/diagnosis, bipolar disorder with comorbidities, rational polypharmacy, gynecomastia, discontinuation syndrome and determination of possible/probable adverse effect causation.

First, this case is consistent with general clinical practice, wherein historical symptoms and even prior diagnoses may not be reported during an appropriate comprehensive initial evaluation and are only retrospectively acknowledged over a naturalistic treatment course. Specifically, this patient described an MDE consistent with a major depressive disorder during his evaluation; he only later revealed earlier onset of bipolar features resulting in the corrected mood disorder diagnosis: bipolar disorder NOS. Delay in reporting both current and historical symptoms may result in inappropriate treatments. Thus, it is important for clinicians to periodically reassess symptom history and review potential alternative diagnoses in treatment-refractory patients, as well as those patients with only partial response to adequate pharmacotherapy.

Second, bipolar disorder outcome is premised on timely and appropriate treatments; however, early-onset bipolar disorders often have both delayed treatment intervention and delayed diagnosis which lead to poorer outcomes, including increased rates of suicidal behaviours. ${ }^{26-28}$ Even in the context of care by specialised mental health services, such delays have been reported for initial presentation with SUD, depression and anxiety disorders. ${ }^{29}$ In this case, initial psychiatric features dated to childhood, bipolar features had been present since age 13, and depressive features since age 16 - the delay to any psychiatric treatment from bipolar disorder onset was 8.5 years, and the delay to appropriate diagnosis and treatment was 9 years.

Third, bipolar disorder is commonly associated with multiple comorbidities. ${ }^{1-10}$ Comorbidity is the rule rather than the exception, as $>75 \%$ of patients with bipolar disorder have at least one lifetime comorbid psychiatric condition, and $>50 \%$ have multiple lifetime comorbid psychiatric conditions. ${ }^{30}$ The findings in this case are consistent with the literature; a summary of all psychiatric comorbidities acknowledged at the time of initial evaluation of this patient and during ensuing treatment over the course of 12 months included GAD, SUD, panic disorder, OCD, ADHD and PTSD. Prevalence rates for comorbid conditions may be similar and/or increased in patients with childhood onset of bipolar disorder compared with lifetime prevalence rates. ${ }^{1-3,7-8,10,31}$ The Course and Outcome of Bipolar Youth (COBY) study noted the following comorbidity prevalence rates: $47.2 \%$ any anxiety disorder, $33.9 \% \mathrm{GAD}, 16.9 \%$ panic disorder, $67.8 \%$ ADHD and $37.3 \%$ any SUD. ${ }^{31}$ The COBY study also confirmed the negative effects of anxiety disorders, SUD and ADHD on acute and longitudinal outcomes for bipolar disorder. ${ }^{31}$

Fourth, rational polypharmacy is frequently required for patients with bipolar disorders with comorbidities. ${ }^{11,12,32}$ As in the present case, when the patient does not present with or acknowledge historical bipolar features, such psychotropic treatment may begin with antidepressants which could worsen acute and longitudinal outcomes; however, the effect on 'switching' and increased mood episodes may be dependent on multiple factors, including antidepressant class, antidepressant treatment duration, bipolar disorder subtype (bipolar I, bipolar II or bipolar NOS) and the presence of rapid cycling. ${ }^{33-35}$ Treatment guidelines recommend initial treatment with standard mood-stabilising psychotropics (lithium, antiepileptic drugs and second-generation antipsychotics), to which would then be added, as required, specific psychotropics to address individual comorbidities. ${ }^{11,12,32,35}$ Non-adherence to psychotropic treatment is multifactorial; however, polypharmacy has an increased risk for drug-drug interactions and adverse effects which may lead to decreased adherence. ${ }^{14,16,36,37}$ Consider that this patient decreased both escitalopram and buspirone by $50 \%$ simply to see whether he would feel better on a lower dose and, by so doing, precipitated a discontinuation syndrome. Since bipolar disorder management requires an appreciation of acute and long-term psychotropic efficacy, safety and tolerability, when treating patients with bipolar disorder with comorbidities, clinicians should also consider which psychotropics may effectively treat multiple conditions while considering both acute and long-term number needed to treat/number needed to harm ratios. ${ }^{12,13,33,35,38}$

Fifth, gynecomastia is a frequent benign finding in men (prevalence range 32-65\%) that significantly increases with BMI $>25 \mathrm{~kg} / \mathrm{m}^{2}{ }^{39}$ Although gynecomastia is predominantly idiopathic, other aetiologies include medication, systemic illness (hepatic, renal, hormonal) and tumors. ${ }^{21,22,39-41}$ This patient had normal standard laboratories and BMI; only his testosterone level was borderline low, for which he briefly received testosterone gel that was discontinued upon development of contralateral gynecomastia - this level returned to normal later in the treatment course. Pertinent to the findings in this case, a PubMed literature review noted the following classes of medications, specific medications and substances as potential factors in the development of gynecomastia - selective serotonin reuptake inhibitors, benzodiazepines, bupropion, marijuana, testosterone, amphetamines and second-generation antipsychotics (aripiprazole has been utilised to reverse gynecomastia). ${ }^{21,22,40-46}$ There are no reported cases of gynecomastia induced by buspirone, lurasidone, lithium, aripiprazole or lamotrigine. One case report describes gynecomastia induced by the anxiolytic tandospirone, a $5-\mathrm{HT}_{1 \mathrm{~A}}$ partial agonist related to buspirone. ${ }^{47}$

Sixth, determination of causation for gynecomastia and mammoplasia in psychiatric patients is especially difficult, as patients rarely volunteer this condition. ${ }^{21,22,40}$ This patient openly addressed his gynecomastia at each session, permitting the development of a timeline based on alterations in psychotropics.

(a) Unilateral gynecomastia first reported - bupropion $300 \mathrm{mg}$ q.a.m., escitalopram $7.5 \mathrm{mg}$ q.a.m., buspirone $15 \mathrm{mg}$ t.i.d., lorazepam $0.5-1.0 \mathrm{mg}$ t.i.d. p.r.n., topical testosterone (3 week course with discontinuation secondary to gynecomastia) and self-medication with daily marijuana

(b) Persistent gynecomastia - bupropion $300 \mathrm{mg}$ q.a.m., buspirone $15 \mathrm{mg}$ b.i.d., lorazepam $0.5-1.0 \mathrm{mg}$ t.i.d. p.r.n., amphetaminedextroamphetamine extended release $10 \mathrm{mg}$ q.a.m. and self-medication with daily marijuana (lurasidone and lithium both utilised briefly with discontinuation secondary to adverse effects)

(c) Minimal gynecomastia without tenderness - buspirone tapered to $15 \mathrm{mg}$ q.a.m., bupropion $300 \mathrm{mg}$ q.a.m., lorazepam $0.5-1.0 \mathrm{mg}$ t.i.d. p.r.n., amphetamine-dextroamphetamine extended release $20 \mathrm{mg}$ q.a.m. and self-medication with daily marijuana (aripiprazole utilised briefly with discontinuation secondary to adverse effects)

(d) Recurrent gynecomastia with tenderness and pain - buspirone increased to $15 \mathrm{mg}$ b.i.d., bupropion $300 \mathrm{mg}$ q.a.m., lorazepam 0.5-1.0 mg t.i.d. p.r.n., amphetamine-dextroamphetamine extended release $20 \mathrm{mg}$ q.a.m. and self-medication with daily marijuana

(e) Persistent gynecomastia - buspirone $15 \mathrm{mg}$ b.i.d., bupropion $300 \mathrm{mg}$ q.a.m., lorazepam $0.5-1.0 \mathrm{mg}$ t.i.d. p.r.n., amphetamine-dextroamphetamine extended release $20 \mathrm{mg}$ q.a.m., lamotrigine $100 \mathrm{mg}$ q.d. and self-medication with daily marijuana

(f) Resolved gynecomastia on physical exam by breast specialist buspirone discontinued, bupropion $300 \mathrm{mg}$ q.a.m., lorazepam $1 \mathrm{mg}$ t.i.d. p.r.n., amphetamine-dextroamphetamine extended release $20 \mathrm{mg}$ q.a.m., lamotrigine $150 \mathrm{mg}$ q.d. and self-medication with daily marijuana

This medication timeline revealed an on/off/on/off titration of buspirone directly correlated to the degree of gynecomastia, with 
ultimate resolution of observable/palpable gynecomastia with total discontinuation of buspirone (a repeat mammogram noted mild bilateral gynecomastia). Although other medications or substances or even hormonal imbalance may have played an additive part in this patient's gynecomastia, buspirone-induced gynecomastia is considered probable by the Naranjo probability scale. ${ }^{48}$

Seventh, antidepressant discontinuation syndromes occur with all classes of antidepressants, with a broad range of symptoms, present even with gradual psychotropic tapering, can be precipitated by psychotropic non-adherence, and may have varying time duration including different durations for specific symptoms. ${ }^{23-25,49}$ In this case, symptoms associated with gradual escitalopram discontinuation are consistent with the above principles. Buspirone withdrawal/discontinuation syndrome has not been previously reported. ${ }^{50-52}$ Further, dental pain has not been previously reported in any psychotropic discontinuation or withdrawal syndrome. As such, buspirone discontinuation syndrome with dental pain represents a case of first impression. Similar to antidepressant discontinuation syndromes, the dental pain had a different time duration compared with other discontinuation features. Based on the medication timeline and the on/off/on/off buspirone titration, the buspirone discontinuation syndrome with dental pain is considered probable by the Naranjo probability scale. ${ }^{48}$

This case has multiple limitations. (a) As a case report $(N=1)$, the findings cannot be generalised. (b) Comprehensive hormonal panels were not assessed during the treatment course beyond thyroid function tests and testosterone levels. Oestrogen balance and hyperprolactinemia are associated with gynecomastia. Baseline hormonal values and assessments throughout the treatment course might have suggested both predisposing hormonal factors and the influence of psychotropic titrations on hormones influencing gynecomastia. (c) No psychotropic blood levels were obtained to address concentration-dependent as opposed to dose-dependent effects of buspirone on gynecomastia. (d) Breast biopsies were not considered clinically indicated and there was no histopathology confirmation of benign gynecomastia. (e) Urine/serum toxicology for cannabinoids and active metabolites was not obtained, which precluded determining whether there was a correlation with gynecomastia. (f) Psychometric scales were not utilized, though such scales can be of benefit in monitoring symptom severity, especially when patients are seen infrequently in follow-up (this patient was followed every 1-3 weeks based on symptom severity and development of adverse effects). (g) As a naturalistic clinical case, for ethical reasons this patient could not be re-challenged or retested for further determinations.

\section{Conclusions}

This case summarises some of the important complexities associated with bipolar disorder treatment: incomplete patient symptom reporting requiring reassessment of historical symptoms during treatment course to ensure appropriate diagnoses; early onset with delayed treatment/diagnosis; frequent comorbidities; rational polypharmacy with adverse effects; and psychotropic non-adherence.

Rational polypharmacy in bipolar disorder with comorbid psychiatric conditions may reveal unusual adverse effects. This report presents a case of first impression for both dose-dependent buspirone-induced gynecomastia and buspirone discontinuation syndrome with a new discontinuation symptom, dental pain. Clinicians and patients should consider these findings to maximise treatment adherence, minimise any unnecessary interventions and address unusual adverse effects. Since patients may not voluntarily disclose specific adverse effects and often do not acknowledge non-adherence, clinician-directed questions are required. ${ }^{16,20,22}$ This case further emphasises the importance of medication and symptom timelines to guide determination of causation for adverse effects. Although findings from this case cannot be generalised, they suggest the need for continued clinician and patient education, as well as the benefit from detailed case reports.

Kenneth R. Kaufman, MD, FRCPsych, DLFAPA, FAES, Departments of Psychiatry Neurology, and Anesthesiology, Rutgers Robert Wood Johnson Medical School, New Brunswick, New Jersey, USA; Ronke Babalola, MD, MPH, Miriam Campeas, BA, Melissa Coluccio, BS, Department of Psychiatry, Rutgers Robert Wood Johnson Medical School, New Brunswick, New Jersey, USA

Correspondence: Kenneth R. Kaufman, Departments of Psychiatry, Neurology, and Anesthesiology, Rutgers Robert Wood Johnson Medical School, 125 Paterson Street, suite \#2200, New Brunswick, New Jersey 08901, USA. Email: kaufmakr@rwjms.rutgers. edu

First received 28 Jul 2017, final revision 8 Nov 2017, accepted 14 Dec 2017

\section{References}

1 Nabavi B, Mitchell AJ, Nutt D. A lifetime prevalence of comorbidity between bipolar affective disorder and anxiety disorders: a meta-analysis of 52 interviewbased studies of psychiatric population. EBioMedicine 2015; 2(10): 1405-19.

2 Preti A, Vrublevska J, Veroniki AA, Huedo-Medina TB, Fountoulakis KN. Prevalence, impact and treatment of generalised anxiety disorder in bipolar disorder: a systematic review and meta-analysis. Evid Based Ment Health 2016; 19(3): 73-81.

3 Amerio A, Stubbs B, Odone A, Tonna M, Marchesi C, Ghaemi SN. The prevalence and predictors of comorbid bipolar disorder and obsessive-compulsive disorder: a systematic review and meta-analysis. J Affect Disord 2015; 186: 99-109.

4 Joslyn C, Hawes DJ, Hunt C, Mitchell PB. Is age of onset associated with severity, prognosis, and clinical features in bipolar disorder? A meta-analytic review. Bipolar Disord 2016; 18(5): 389-403.

5 Das A. Anxiety disorders in bipolar I mania: prevalence, effect on illness severity, and treatment implications. Indian J Psychol Med 2013; 35(1): 53-9.

6 Kim SW, Berk L, Kulkarni J, Dodd S, de Castella A, Fitzgerald PB, et al. Impact of comorbid anxiety disorders and obsessive-compulsive disorder on 24-month clinical outcomes of bipolar I disorder. J Affect Disord 2014; 166: 243-8.

7 Hunt GE, Malhi GS, Cleary M, Lai HM, Sitharthan T. Comorbidity of bipolar and substance use disorders in national surveys of general populations, 19902015: systematic review and meta-analysis. J Affect Disord 2016; 206: 321-30.

8 Hunt GE, Malhi GS, Cleary M, Lai HM, Sitharthan T. Prevalence of comorbid bipolar and substance use disorders in clinical settings, 1990-2015: systematic review and meta-analysis. J Affect Disord 2016; 206: 331-49.

9 Robinson J, Sareen J, Cox BJ, Bolton J. Self-medication of anxiety disorders with alcohol and drugs: results from a nationally representative sample. J Anxiety Disord 2009; 23(1): 38-45.

10 Perroud N, Cordera P, Zimmermann J, Michalopoulos G, Bancila V, Prada P, et al. Comorbidity between attention deficit hyperactivity disorder (ADHD) and bipolar disorder in a specialized mood disorders outpatient clinic. $J$ Affect Disord 2014; 168: 161-6.

11 Singh JB, Zarate Jr CA. Pharmacological treatment of psychiatric comorbidity in bipolar disorder: a review of controlled trials. Bipolar Disord 2006; 8(6): 696-709.

12 Schaffer A, McIntosh D, Goldstein BI, Rector NA, McIntyre RS, Beaulieu S, et al. The CANMAT task force recommendations for the management of patients with mood disorders and comorbid anxiety disorders. Ann Clin Psychiatry 2012; 24(1): 6-22

13 Ketter TA, Miller S, Dell'Osso B, Calabrese JR, Frye MA, Citrome L. Balancing benefits and harms of treatments for acute bipolar depression. I Affect Disord 2014; 169(Suppl 1): S24-33.

14 Baldessarini R, Henk H, Sklar A, Chang J, Leahy L. Psychotropic medications for patients with bipolar disorder in the United States: polytherapy and adherence. Psychiatr Serv 2008; 59(10): 1175-83.

15 Serna MC, Real J, Cruz I, Galván L, Martin E. Monitoring patients on chronic treatment with antidepressants between 2003 and 2011: analysis of factors associated with compliance. BMC Public Health 2015; 15: 1184.

16 Mitchell AJ. Depressed patients and treatment adherence. Lancet 2006; 367 (9528): 2041-3. 
17 Keck Jr PE, McElroy SL, Strakowski SM, Bourne ML, West SA. Compliance with maintenance treatment in bipolar disorder. Psychopharmacol Bull 1997; 33(1): 87-91.

18 Fountoulakis KN, Vieta E, Young A, Yatham L, Grunze $\mathrm{H}$, Blier $\mathrm{P}$, et al. The International College of Neuropsychopharmacology (CINP) treatment guidelines for bipolar disorder in adults (CINP-BD-2017), part 4: unmet needs in the treatment of bipolar disorder and recommendations for future research. Int J Neuropsychopharmacol 2017; 20(2): 196-205.

19 Rosenberg KP, Bleiberg KL, Koscis J, Gross C. A survey of sexual side effects among severely mentally ill patients taking psychotropic medications: impact on compliance. J Sex Marital Ther 2003; 29(4): 289-96.

20 Kaufman KR, Wong S, Sivaraaman K, Anim C, Delatte D. Epilepsy and AEDinduced decreased libido - the unasked psychosocial comorbidity. Epilepsy Behav 2015; 52(Pt A): 236-8.

21 Amsterdam JD, Garcia-Espana F, Goodman D, Hooper M, Hornig-Rohan M Breast enlargement during chronic antidepressant therapy. J Affect Disord 1997; 46: 151-6.

22 Kaufman KR, Podolsky D, Greenman D, Madraswala R. Antidepressant-selective gynecomastia. Ann Pharmacother 2013; 47(1): e6.

23 Harvey BH, Slabbert FN. New insights on the antidepressant discontinuation syndrome. Hum Psychopharmacol 2014; 29(6): 503-16.

24 Fava GA, Gatti A, Belaise C, Guidi J, Offidani E. Withdrawal symptoms after selective serotonin reuptake inhibitor discontinuation: a systematic review. Psychother Psychosom 2015; 84(2): 72-81.

25 Narayan V, Haddad PM. Antidepressant discontinuation manic states: a critical review of the literature and suggested diagnostic criteria. J Psychopharmacol 2011; 25(3): 306-13.

26 Post RM, Leverich GS, Kupka RW, Keck Jr PE, McElroy SL, Altshuler LL, et al. Early-onset bipolar disorder and treatment delay are risk factors for poor outcome in adulthood. J Clin Psychiatry 2010; 71(7): 864-72.

27 Drancourt N, Etain B, Lajnef M, Henry C, Raust A, Cochet B, et al. Duration of untreated bipolar disorder: missed opportunities on the long road to optimal treatment. Acta Psychiatr Scand 2013; 127(2): 136-44.

28 Altamura AC, Dell'Osso B, Berlin HA, Buoli M, Bassetti R, Mundo E. Duration of untreated illness and suicide in bipolar disorder: a naturalistic study. Eur Arch Psychiatry Clin Neurosci 2010; 260(5): 385-91.

29 Patel R, Shetty H, Jackson R, Broadbent M, Stewart R, Boydell J, et al. Delays before diagnosis and initiation of treatment in patients presenting to mental health services with bipolar disorder. PLOS One 2015; 10(5): e0126530.

30 Merikangas KR, Jin R, He JP, Kessler RC, Lee S, Sampson NA, et al. Prevalence and correlates of bipolar spectrum disorder in the world mental health survey initiative. Arch Gen Psychiatry 2011; 68(3): 241-51.

31 Yen S, Stout R, Hower H, Killam MA, Weinstock LM, Topor DR, et al. The influence of comorbid disorders on the episodicity of bipolar disorder in youth. Acta Psychiatr Scand 2016; 133(4): 324-34.

32 McIntyre RS, Konarski JZ, Yatham LN. Comorbidity in bipolar disorder: a framework for rational treatment selection. Hum Psychopharmacol 2004; 19(6): 369-86.

33 Malhi GS, Bargh DM, McIntyre R, Gitlin M, Frye MA, Bauer M, et al. Balanced efficacy, safety, and tolerability recommendations for the clinical management of bipolar disorder. Bipolar Disord 2012; 14(Suppl 2): 1-21.
34 Sidor MM, MacQueen GM. An update on antidepressant use in bipolar depression. Curr Psychiatry Rep 2012; 14(6): 696-704.

35 Freeman MP, Freeman SA, McElroy SL. The comorbidity of bipolar and anxiety disorders: prevalence, psychobiology, and treatment issues. J Affect Disord 2002; 68(1): 1-23.

36 Malhi GS, Bargh DM, Cashman E, Frye M, Gitlin M. The clinical management of bipolar disorder complexity using a stratified model. Bipolar Disord 2012; 14 (Suppl 2): 66-89.

37 Mago R, Borra D, Mahajan R. Role of adverse effects in medication nonadherence in bipolar disorder. Harv Rev Psychiatry 2014; 22(6): 363-6.

38 Kaufman KR. Antiepileptic drugs in the treatment of psychiatric disorders. Epilepsy Behav 2011; 21(1): 1-11.

39 Rahmani S, Turton P, Shaaban A, Dall B. Overview of gynecomastia in the modern era and the Leeds Gynaecomastia Investigation algorithm. Breast J 2011; 17 (3): 246-55.

40 Bowman JD, Kim H, Bustamante JJ. Drug-induced gynecomastia. Pharmacotherapy 2012; 32(12): 1123-40.

41 Damsa C, Bumb A, Bianchi-Demicheli F, vidailhet P, Sterck R, Andreoli A, et al. 'Dopamine-dependent' side effects of selective serotonin reuptake inhibitors: $a$ clinical review. J Clin Psychiatry 2004; 65(8): 1064-8.

42 Bergman D, Futterweit W, Segal R, Sirota D. Increased oestradiol in diazepam related gynaecomastia. Lancet 1981; 2(8257): 1225-6.

43 Jelenkovic AV, Macukanovic-Golubovic LDj. Diazepam-associated gynecomastia. Ann Pharmacother 2005; 39(1): 201.

44 Allen RC, Wallace AM, Royce M. Marinol-induced gynecomastia: a case report. Am J Med 2007; 120(10): e1.

45 Basaria S. Androgen abuse in athletes: detection and consequences. J Clin Endocrinol Metab 2010; 95(4): 1533-43.

46 Boggs DL, Ranganathan $M$, Boggs AA, Bihday CM, Peluse BE, D'Souza DC. Treatment of hyperprolactinemia and gynecomastia with adjunctive aripiprazole in 2 men receiving long-acting injectable antipsychotics. Prim Care Companion CNS Disord 2013; 15(4): 13101519.

47 Kaneda $Y$, Morimoto T, Fujii A. Gynecomastia induced by treatment with tandospirone, a 5-HT1A agonist. J Psychiatry Neurosci 2001; 26(2): 152-3.

48 Naranjo CA, Busto U, Sellers EM, Sandor P, Ruiz I, Roberts EA, et al. A method for estimating the probability of adverse drug reactions. Clin Pharmacol Ther 1981; 30(2): 239-45.

49 Hosenbocus S, Chahal R. SSRIs and SNRIs: a review of the discontinuation syndrome in children and adolescents. J Can Acad Child Adolesc Psychiatry 2011; 20(1): 60-7.

50 Feighner JP, Boyer WF. Serotonin-1A anxiolytics: an overview. Psychopathology 1989; 22(Suppl 1): 21-6.

51 Taylor DP. Serotonin agents in anxiety. Ann N Y Acad Sci 1990; 600: 545-56.

52 Howland RH. Potential adverse effects of discontinuing psychotropic drugs: part 2: antidepressant drugs. J Psychosoc Nurs Ment Health Serv 2010; 48(7): $9-12$. 